\title{
E-Z Notation
}

National Cancer Institute

\section{Source}

National Cancer Institute. E-Z Notation. NCI Thesaurus. Code C92192.

A system for naming the stereoisomers of a molecule. The configuration at each double bond is determined by the priority of the side groups and whether the highest priority groups are on the same or opposite side. A Z indicates that the highest-priority side groups are on the same side and an E indicates that the highest-priority side groups are on opposite sides. Priority is given to the bonded atom with the higher atomic number. 